\title{
Pedunculated Ampullary Adenoma Recurs as Carcinoma After Ampullectomy
}

\author{
Joon Han Jeon, MD, Dae Young Cheung, MD, Kwang Yeol Paik, MD, Seong Jin Lee, MD, \\ Hyun Jin Kim, MD, Hye Kang Kim, MD, Hyung Jun Cho, MD, Wook Kim, MD \\ Department of Gastroenterology, the Catholic University of Korea, College of Medicine, Seoul, South Korea \\ (Drs. Jeon, Cheung, Lee, H. J. Kim, H. K. Kim, Cho). \\ Department of Surgery, The Catholic University of Korea, College of Medicine, Seoul, South Korea (Drs. Paik, W. Kim).
}

\begin{abstract}
Introduction: Pedunculated ampullary adenoma is a rare periampullary tumor. There is still controversy regarding whether to perform local ampullectomy or radical resection for adenomas with severe dysplasia.

Case Description: A 57-year-old man was diagnosed with a large, long pedunculated ampullary tumor arising from the second portion of the duodenum. Histologic examination of the biopsy specimen showed a tubular adenoma with low-grade dysplasia. Laparoscopic transduodenal ampullectomy was conducted, and the tumor was removed surgically with the grossly sufficient free margin. The final histologic examination of the resected tumor showed high-grade dysplasia. Two months later, the mass was found to have recurred and proved to be well-differentiated adenocarcinoma. Finally, the patient underwent pancreaticoduodenectomy.

Discussion: Local resection may be acceptable for benign ampullary tumors. However, radical resection should be considered for suspected adenocarcinoma. It comprises a large tumor size, the presence of intraductal extension, and findings of preprocedural high-grade dysplasia. The decision to perform local resection for large tumors should be made carefully because most large tumors are proved to be cancer, and local resection plays a limited role in ampullary cancer. Radical resection should be considered for suspected adenocarcinoma, especially in cases of large tumors or in the presence of preprocedural high-grade dysplasia.
\end{abstract}

Key Words: Ampullary neoplasm, Ampullary adenoma, Ampullary carcinoma, Pedunculated ampullary adenoma, Ampullectomy.

Citation Jeon JH, Cheung DY, Paik KY, Lee SJ, Kim HJ, Kim HK, Cho HJ, Kim W. Pedunculated ampullary adenoma recurs as carcinoma after ampullectomy. CRSLS e2014.00356. DOI: 10.4293/CRSLS.2014.00356.

Copyright (C) 2014 SLS This is an open-access article distributed under the terms of the Creative Commons Attribution-Noncommercial-ShareAlike 3.0 Unported license, which permits unrestricted noncommercial use, distribution, and reproduction in any medium, provided the original author and source are credited.

Address correspondence to: Dae Young Cheung, MD, The Catholic University of Korea, Yeouido St. Mary Hospital, Yeongdeungpo-gu, 63-ro 10, Seoul, 150-713,

South Korea. Telephone: +82-2-3779-1519, Fax: +82-2-3779-1331, E-mail: adagio@catholic.ac.kr

\section{INTRODUCTION}

Ampullary carcinoma is uncommon among gastrointestinal malignancies, and pedunculated ampullary adenoma is a rare periampullary tumor. ${ }^{1}$ Pancreaticoduodenectomy is the treatment of choice for invasive ampullary carcinomas; however, there is still controversy regarding whether to perform local ampullectomy or radical resection for adenomas with severe dysplasia. ${ }^{2-6}$ This is because of the increased tendency for these lesions to recur after local ampullectomy. ${ }^{7,8}$ We report a case of pedunculated ampullary carcinoma that recurred after local resection of ampullary adenoma.

\section{CASE REPORT}

A 57-year-old man who was generally healthy except for an infrequently occurring indigestive feeling visited a regional hospital to participate in the Korean national cancer screening program. Esophagogastroduodenoscopy (EGD) showed a pedunculated mass of the duodenum, and he was referred to our institution for further investigation. He had no specific medical or family history. On physical examination, the patient was found to have no palpable mass. The results of laboratory studies and tumor markers including carcino embrionic antigen and carbo- 
hydrate antigen 19-9 were within normal ranges, except for an alkaline phosphatase level of $737 \mathrm{U} / \mathrm{L}$ and $\gamma$-glutamyl transpeptidase level of $352 \mathrm{U} / \mathrm{L}$. Repeated EGD showed a pedunculated villous-appearing mass with a diameter of $45 \mathrm{~mm}$ in the second portion of the duodenum (Figure 1). The length of stalk was estimated to be about $50 \mathrm{~mm}$. Microscopic examination of a forceps biopsy specimen showed tubular adenoma with low-grade dysplasia. On an enhanced computed tomography scan, a duodenal mass was noted; it appeared moderately attenuated, measured $52 \times 32 \times 26 \mathrm{~mm}$, and showed no subserosal involvement (Figure 2).

Because the tumor had a sufficiently long pedicle, endoscopic resection was attempted as the primary treatment strategy with an operative team backup in the operating room. However, the massive tumor hindered adequate ensnaring, and the voluminous pedicle implied the presence of massive bleeding and incomplete resection. The treatment strategy was changed to laparoscopic resection onsite. After the location of the tumor was indentified with endoscopic light illumination guidance, laparoscopic duodenotomy was performed in the second portion of the duodenum longitudinally. The pedunculated adenoma bulged out through the incised wall and was pulled out with a laparoscopic spatula. A long pedicle connected to the tumor was identified and cut using an endoscopic
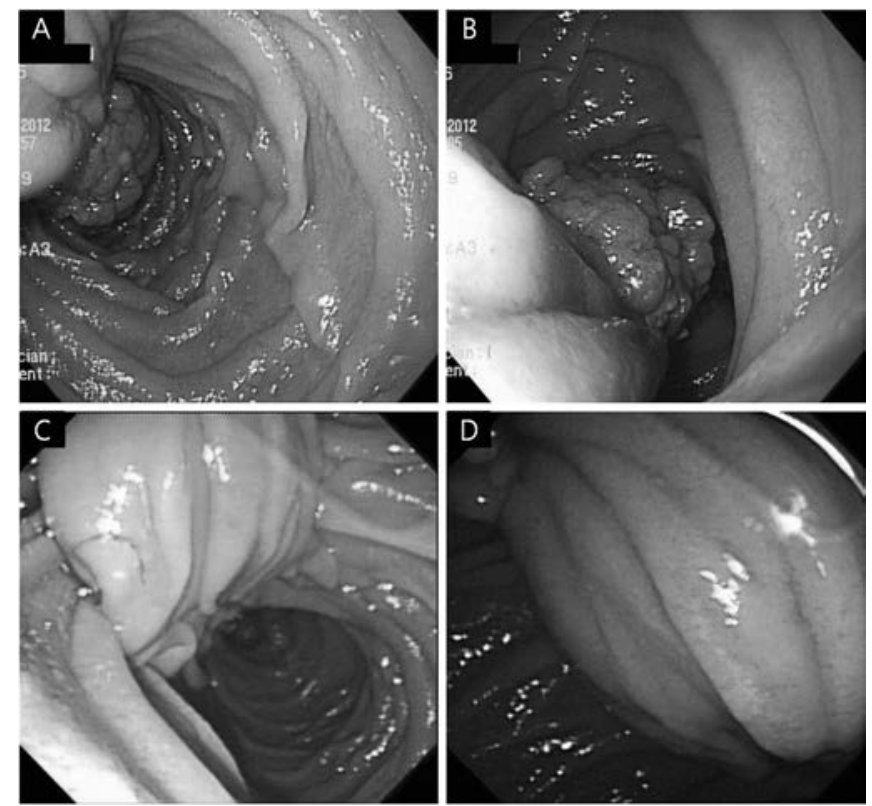

Figure 1. Endoscopic findings in duodenum. A and B, Polypoid mass with long stalk in duodenum. $\mathrm{C}$ and $\mathrm{D}$, The base of the long stalk originated from the inferior descending angle of the duodenum through an endoscopic view.
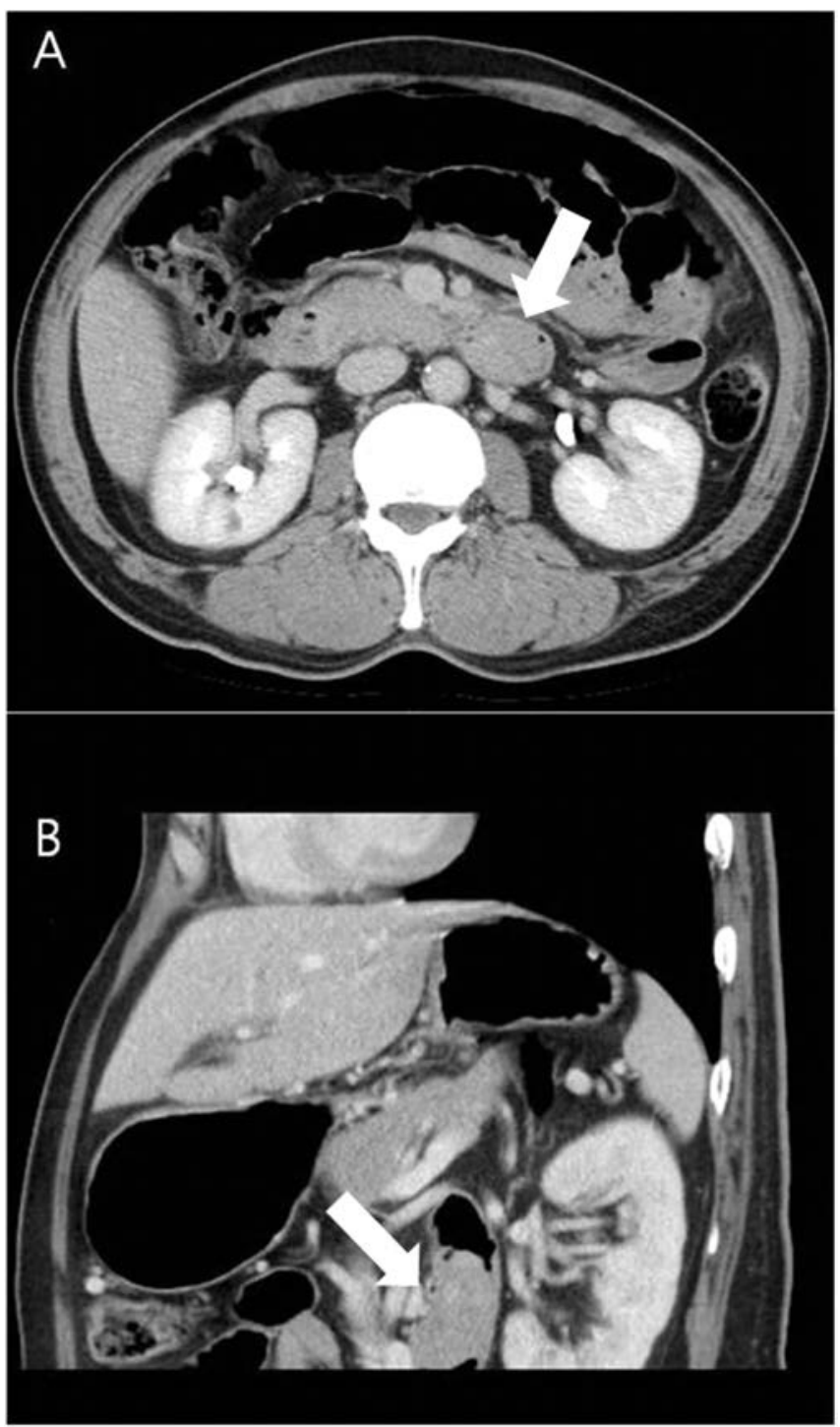

Figure 2. The tumor measured $52 \times 32 \times 26 \mathrm{~mm}$. A, An abdominal enhanced abdominal computed tomography scan showed a moderately attenuated mass in the third portion of the duodenum (arrow). B, A sagittal image also showed a mass with homogeneous and moderate attenuation (arrow).

stapling system. The pedunculated tumor was resected with a 2.0-cm margin of safety (Figure 3A). The resected tumor measured $57 \times 46 \mathrm{~mm}$. After duodenotomy, the opening was repaired by laparoscopic suturing in the horizontal direction. EGD was performed to ascertain the condition of the duodenotomy repair. With careful endoscopic examination, we could not identify the ampulla of Vater. Under the given circumstances, the ampulla of Vater was suspected to be included in the resected tumor. Thorough examination of the resected mass 


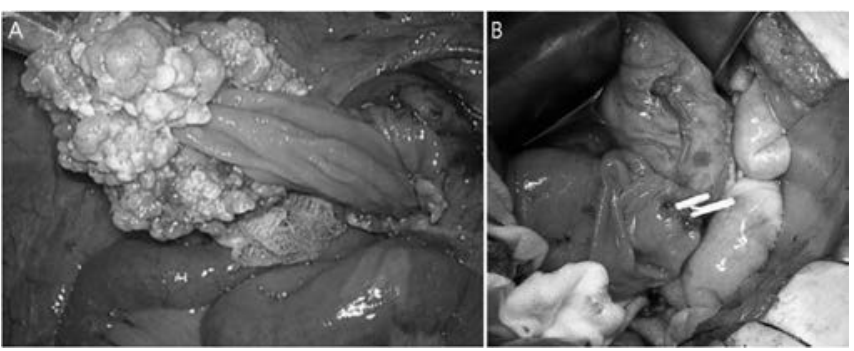

Figure 3. Operative findings. A, Laparoscopy showed a $57 \times$ $46-\mathrm{mm}$ pedunculated tumor in the ampulla of Vater with a long stalk. B, Two plastic stents were placed in the bile and pancreatic ducts through laparotomy.

showed a small opening inside. Thus we performed cholecystectomy so that we could perform cholangiography. Intraoperative cholangiography via the cystic duct visualized an abrupt cutoff sign of the distal common bile duct. We decided that conversion to open laparotomy was necessary, and the duodenotomy was reopened. The closed stump of resected pedicle on the duodenal posterior wall was also reopened. After careful examination, the bile duct and pancreatic juice were identified and $7 \mathrm{~F}$ $5.0-\mathrm{cm}$ plastic tubes were inserted into the ducts. Clear bile and pancreatic duct flowed through the tubes. Circumferential suturing was performed to fix the ducts into the duodenal wall by using fine silk. Tubes were fixed to the ducts by knotting the suture silk (Figure 3B). The operation was completed after stent patency was ascertained. Histologic examination showed that the resected tumor was composed of tubular adenoma with high-grade dysplasia, and the resection margin was clear. The patient was discharged 10 days later without complication, and the biliopancreatic plastic stents were expected to be removed spontaneously with time.

The patient returned for a routine checkup 2 months later. He reported no specific complaints, but laboratory tests showed an elevated alkaline phosphatase level, $354 \mathrm{U} / \mathrm{L}$, and $\gamma$-glutamyl transpeptidase level of $103 \mathrm{U} / \mathrm{L}$. To assess the condition of the bile duct tube, EGD was performed. The pancreatic duct tube had disappeared, and the bile duct tube remained, with a $2.0-\mathrm{cm}$ polypoid mass encircling the tube (Figure 4). Biopsy findings showed a welldifferentiated adenocarcinoma. The patient was readmitted, and pancreaticoduodenectomy was scheduled for curative resection. An abdominal computed tomography scan showed a homogeneous attenuated mass with a diameter of $23 \times 20 \mathrm{~mm}$ in the second portion of the duodenum without abnormal lymphadenopathy or ascites. Pancreaticoduodenectomy was performed about 2 months after the initial conversion to ampullectomy and

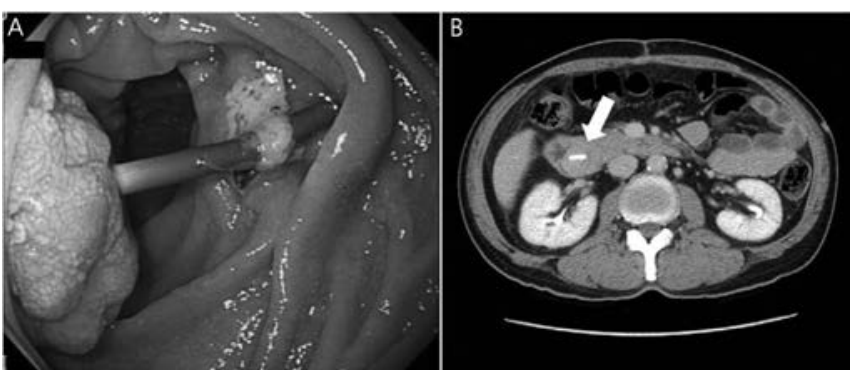

Figure 4. Follow-up endoscopic findings. A, A 20-mm polypoid mass encircling a drainage tube was seen. B, A follow-up computed tomography scan showed a $23 \times 20-$ mm homogeneous attenuated mass encircling the stent in the duodenum. (A drainage tube appears as radio-opaque bar in CT, arrow).

completed as usual without immediate complications or technical difficulties. No abnormal lymph nodes were found at surgery. The resected tumor measured $30 \times 25 \times$ $15 \mathrm{~mm}$, and the distal common bile was intact. Histologic examination showed a tubular, well-differentiated adenocarcinoma invading the duodenal wall and the intraductal structure of the bile duct (Figure 5). The resection margin was clear, and no regional lymph node involvement was reported ( 0 of 16 nodes). The postoperative course of the patient was uneventful, with regular EGD follow-up visits showing no recurrence.

\section{DISCUSSION}

Whether ampullary tumors are best treated surgically by pancreaticoduodenectomy or local ampullectomy remains controversial. Histologic examination, tumor size, and tumor extent are known to be important factors when deciding the treatment strategy. Radical surgery provides reliable curative resection; however, the cost of pancreaticoduodenectomy often outweighs the benefit for limited disease. Although guidelines and consensus statements are still being developed, general practical directions agree that small and low-grade tumors of the ampulla can be treated with local excision modalities. Such modalities consist of endoscopic ampullectomy and surgical transduodenal ampullectomy. The definition of a small adenoma is not concrete. Considering the endoscopic loop snare size, $2.0 \mathrm{~cm}$ is the possible limit for safe and proper ensnaring for endoscopic resection. The literature supports this idea and suggests that endoscopic ampullectomy can be safely used for ampullary adenomas $<2.0 \mathrm{~cm}$ in size and without extra-ampullary extension. ${ }^{9-11}$ The success rate for endoscopic ampullectomy ranges from $70 \%$ to $90 \% .{ }^{3,10}$ Regarding tumor histology, a recent retrospective review reported that high-grade intraepithelial 
Pedunculated Ampullary Adenoma Recurs as Carcinoma After Ampullectomy, Jeon JH et al.

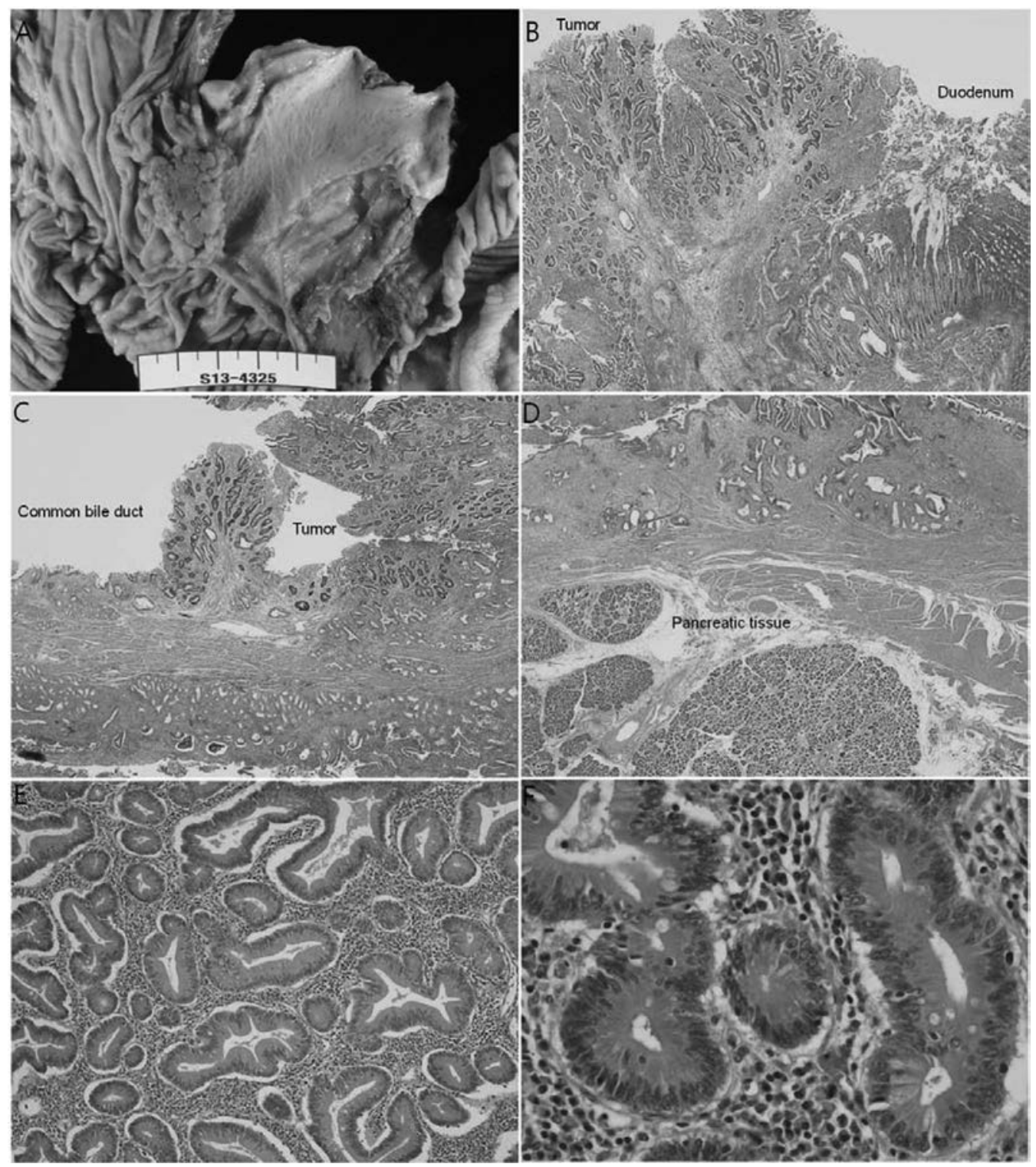

Figure 5. Specimen and histologic findings. A, Gross appearance. B and C, Tumor invasion into duodenal wall and distal common bile duct (hematoxylin-eosin stain, original magnification $\times 12.5$ ). D, The pancreas was free from tumor (hematoxylin-eosin stain, original magnification $\times 12.5$ ). E and F, Representative tissue showing well-differentiated adenocarcinoma (hematoxylin-eosin stain, original magnification $\times 100$ and $\times 400$, respectively).

neoplasias or in situ tumors of the ampulla of Vater without lymphovascular invasion and lymph node metastasis were successfully treated with endoscopic ampullectomy. ${ }^{6}$ In this study even focal T1 cancers without lymphovascular and lymph node involvement were treated with endoscopic ampullectomy, and there were no cancer recurrences or cancer-related deaths. However, concern regarding endoscopic ampullectomy has been noted because there is a high rate of tumor recurrence on follow- up; rates of $8 \%^{10}$ to $42 \%^{12}$ have been reported. In a Korean study, the larger size of an adenoma was proved to correlate with a higher conversion rate of cancer diagnosis. ${ }^{13}$ The authors suggested that ampullary adenoma preoperatively diagnosed with high-grade dysplasia or measuring $>1.5 \mathrm{~cm}$ in size should not be managed with endoscopic ampullectomy because of high recurrence rates, up to $80 \%$. The possible reason for the high tumor recurrence rates after endoscopic ampullectomy may lie in the pos- 
sible inaccuracy of preoperative forceps biopsy diagnosis and insufficient safety margins of the resection procedure. An ampullary adenoma diagnosed with forceps biopsy turns out to be adenocarcinoma after resection in 5\% to $20 \%$ of cases. ${ }^{2}$ To secure the curative resection, radical surgery has been advocated for ampullary tumors, even including adenomas. Especially for high-grade adenomas, some experts recommend radical surgery because of higher recurrence rates and frequent coexistence of malignancy at the time of diagnosis. The limited accuracy of forceps biopsy is partly responsible for a high incidence of malignancy in ampullary villous tumors, with rates of 5\% to $30 \%$. High-grade dysplasia on endoscopic biopsy examination and ductal dilatation are significant predictors of ampullary cancer. ${ }^{14}$ Thus, if these findings present, radical resection should be considered. However, the postoperative morbidity rate for radical surgery is estimated at up to $35 \%$ to $65 \%$ and the mortality rate reaches $10 \%$, and these rates should be taken into consideration when the treatment strategy is determined. ${ }^{15}$

Transduodenal ampullectomy can be an intermediate choice between endoscopic ampullectomy and radical surgery. Transduodenal ampullectomy provides a visually adequate operation field, and the safety margins can be more easily obtained. A tumor measuring $<3.0 \mathrm{~cm}$ can be effectively treated with transduodenal ampullectomy, provided that the tumor invasion is confined to the submucosa. ${ }^{16}$ Compared with radical surgery, transduodenal ampullectomy resulted in comparable oncologic outcomes and better clinical outcomes in terms of morbidity and mortality rates. Significant postoperative complications developed in $50 \%$ of Whipple operation cases and $14 \%$ of transduodenal ampullectomy cases. The mortality rate was $8 \%$ in Whipple operation cases, but there were no deaths in transduodenal ampullectomy cases. Another advantage of transduodenal ampullectomy lies in the availability of intraoperative frozen-section histologic examination in the operating room. We have found that frozen-section histologic examination is very helpful in deciding whether immediate intraoperative conversion to pancreaticoduodenectomy should be performed. It can prevent unnecessary pancreaticoduodenectomy in patients with benign tumors of the ampulla of Vater..$^{15,17,18}$ In our patient, intraoperative frozen-section histologic examination showed high-grade dysplasia, and a Whipple operation was not performed during the initial operation. However, the patient presented with tumor recurrence at the bile and pancreatic duct tubing site, and adenocarcinoma was proved by histologic analysis on follow-up endoscopy. A possible explanation for the short-term de- velopment of adenocarcinoma at the margin-free resection site is as follows: an adenoma with high-grade dysplasia developed in the ampulla of Vater; the adenoma grew and created a massive tumor; the adjacent duodenal mucosa was stretched and elongated because of the weight of the tumor; the stretched mucosa created a long pedicle; the adenoma also extended along the bile duct; gross examination failed to recognize the presence of the adenoma inside the duct; operative manipulation of the tumor and pedicle may have resulted in inadequate conditions inside the ducts.

Tumor morphology is another factor in the treatment decision. Most ampullary adenomas and early cancers are sessile in appearance. Pedunculated ampullary adenoma is extremely rare, and we found just 1 case report in the literature. ${ }^{19}$ In this report the pedunculated ampullary adenoma measured $50 \mathrm{~mm}$ and was resected successfully with snare polypectomy. With Experiences in colon polyps, pedunculated adenomas are shown to have better oncologic behavior than sessile adenoma and ensnaring at the stalk makes polypectomy easy irrespective of the tumor size. Therefore the size limitation for ampullary tumors should be separately considered for sessile and pedunculated tumors. If the snare loop can be lodged at the stalk, a pedunculated tumor of any size can be resected properly with sufficient safety margins. Expert consensus recommended endoscopic resection for tumors $<4.0$ to $5.0 \mathrm{~cm}$ in size with no evidence of intraductal growth and coexisting malignancy by endoscopic findings. ${ }^{15,17,20,21}$

One point endoscopists have to keep in mind is that, to provide all necessary procedures along with endoscopic resection, the identification and localization of the ampullary opening are essential. An ampullary adenoma with a pedicle requires additional procedures after resection, including bile and pancreatic duct drainage. In our case, preoperative evaluation failed to identify the location of the ampullary opening, and the consequence resulted in conversion to open laparotomy ampullectomy.

In conclusion, a careful examination is required before beginning treatment of a duodenal pedunculated mass. The decision regarding the treatment modalities for a periampullary tumor should be made by taking into consideration the histologic findings, size and morphologic characteristics of the tumor, and presence of intraductal growth. In the presence of a pedicle, surgeons may favor local resection; however, a large tumor may extend along the ductal system inside the pedicle and have a high possibility of malignancy. Radical resection remains the standard curative procedure for suspected or confirmed 
adenocarcinoma. ${ }^{8}$ For presumed benign ampullary tumors on endoscopic biopsy, transduodenal ampullectomy can serve as an intermediate treatment option before radical resection. ${ }^{18}$ The decision to perform local resection for large tumors should be made carefully because most large tumors are proved to be cancer, and local resection plays a limited role in ampullary cancer. ${ }^{13,22}$ Accurate intraoperative frozen section is indispensable and close follow-up is warranted because of the risk of unrecognized residual tumor and high recurrence rate.

\section{References:}

1. El Hajj II, Coté GA. Endoscopic diagnosis and management of ampullary lesions. Gastrointest Endosc Clin N Am. 2013;23: 95-109.

2. Posner S, Colletti L, Knol J, et al. Safety and long-term efficacy of transduodenal excision for tumors of the ampulla of Vater. Surgery. 2000;128:694-701.

3. Salmi S, Ezzedine S, Vitton V, et al. Can papillary carcinomas be treated by endoscopic ampullectomy? Surg Endosc. 2012;26: 920-925.

4. Winter JM, Cameron JL, Olino K, et al. Clinicopathologic analysis of ampullary neoplasms in 450 patients: implications for surgical strategy and long-term prognosis. J Gastrointest Surg. 2010;14:379-387.

5. Yoon YS, Kim SW, Park SJ, et al. Clinicopathologic analysis of early ampullary cancers with a focus on the feasibility of ampullectomy. Ann Surg. 2005;242:92-100.

6. Yoon SM, Kim MH, Kim MJ, et al. Focal early stage cancer in ampullary adenoma: surgery or endoscopic papillectomy? Gastrointest Endosc. 2007;66:701-707.

7. Demetriades H, Zacharakis E, Kirou I, et al. Local excision as a treatment for tumors of ampulla of Vater. World J Surg Oncol. 2006; $4: 14$.

8. Chini P, Draganov PV. Diagnosis and management of ampullary adenoma: the expanding role of endoscopy. World $J$ Gastrointest Endosc. 2011;3:241-247.

9. Bassan M, Bourke M. Endoscopic ampullectomy: a practical guide. J Interv Gastroenterol. 2012;2:23-30.
10. Catalano MF, Linder JD, Chak A, et al. Endoscopic management of adenoma of the major duodenal papilla. Gastrointest Endosc. 2004;59:225-232.

11. Ridtitid W, Tan D, Schmidt SE, et al. Endoscopic papillectomy: risk factors for incomplete resection and recurrence during long-term follow-up. Gastrointest Endosc. 2014;79:289-296.

12. Galandiuk S, Hermann RE, Jagelman DG, et al. Villous tumors of the duodenum. Ann Surg. 1988;207:234-239.

13. Kim JH, Kim JH, Han JH, et al. Is endoscopic papillectomy safe for ampullary adenomas with high-grade dysplasia? Ann Surg Oncol. 2009;16:2547-2554.

14. Kim HN, Kim KM, Shin JU, et al. Prediction of carcinoma after resection in subjects with ampullary adenomas on endoscopic biopsy. J Clin Gastroenterol. 2013;47:346-351.

15. Adler DG, Qureshi W, Davila R, et al. The role of endoscopy in ampullary and duodenal adenomas. Gastrointest Endosc. 2006; 64:849-854.

16. Rattner DW, Fernandez-del Castillo C, Brugge WR, et al. Defining the criteria for local resection of ampullary neoplasms. Arch Surg. 1996;131:366-371.

17. Tran TC, Vitale GC. Ampullary tumors: endoscopic versus operative management. Surg Innov. 2004;11:255-263.

18. Kim J, Choi SH, Choi DW, et al. Role of transduodenal ampullectomy for tumors of the ampulla of Vater. J Korean Surg Soc. 2011;81:250-256.

19. Shiba H, Misawa T, Wakiyama S, et al. Pedunculated early ampullary carcinoma treated by ampullectomy: report of a case. J Gastrointest Cancer. 2010;41:138-140.

20. Cheng CL, Sherman S, Fogel EL, et al. Endoscopic snare papillectomy for tumors of the duodenal papillae. Gastrointest Endosc. 2004;60:757-764.

21. Okamoto Y, Fujii M, Tateiwa S, et al. Pedunculated early carcinoma of supra-ampullary duodenum presenting as acute pancreatitis. Int J Gastrointest Cancer. 2002;31:185-189.

22. Lindell G, Borch K, Tingstedt B, et al. Management of cancer of the ampulla of Vater: does local resection play a role? Dig Surg. 2003;20:511-515. 